\title{
Image compression using linear prediction operator of wavelet coefficients
}

\author{
S. Selvarajan ${ }^{1^{*}}$, N. D. Kodikara ${ }^{2}$ and G. D. S. P. Wimalaratne ${ }^{3}$ \\ 1 Department of Physical Science, Faculty of Applied Science, Vavuniya Campus of the University of Jaffna, Park Road, Vavuniya. \\ 2 Department of Information Systems Engineering, University of Colombo School of Computing, 35, Reid Avenue, Colombo 7. \\ 3 Department of Communication and Media Technology, University of Colombo School of Computing, 35, Reid Avenue, Colombo 7.
}

\begin{abstract}
In this paper, we propose a new prediction operator called the linear prediction operator. The linear prediction operator is based on the explicit model of statistical relationship of the high-frequency coefficients between the different frequency sub-bands and the scaled similarity of the highfrequency coefficients with respect to their orientations. This scaled similarity is exploited to approximate the functions in different resolutions to achieve high compression ratio.
\end{abstract}

Keywords: Fractal coding, haar transform, multi resolution analysis, nonlinear approximation, zero-tree wavelet.

\section{INTRODUCTION}

The objective of lossy image compression is to store image data efficiently by reducing the redundancy of image content and discarding unimportant information, while keeping the quality of the image acceptable. Typically, images contain strong geometric features, such as edges, that impose a structure on pixel values. Much of the information in an image is communicated by the edges of the object and these edges generally contain a great deal of high-frequency energy.

The multiresolution representation of a function can be represented by compactly supported wavelet basis function. The advantage of wavelet transform of an image is that most of the energy is concentrated in few significant coefficients. The fine detail coefficients representing the information of an image are sparsely distributed within the frequency sub-bands. Thus, much of the information is contained in representations of the positions of those few coefficients corresponding to significant anomalies. Many methods of lowering such cost of encoding the position information associated with the significant coefficients have been proposed, based on the idea of prediction across frequency bands which exploits the self-similar, hierarchal nature of wavelet transform. A notable breakthrough was the introduction of Embedded Zero-tree Wavelet (EZW) coding by Shapiro ${ }^{1}$. Based on the concept of EZW, a remarkable approach Set Partitioning in Hierarchial Trees (SPIHT) was introduced by Said and Perlman ${ }^{2}$. It has been extensively studied by Cohen et al. ${ }^{3,4}$.

Rinaldo and Calvagno ${ }^{5}$ proposed an algorithm using the self-similarity in pyramid sub-band decomposition in fractal coding methods. The pyramid data structures suffer from the difficulty that the data at separate levels are correlated. There is no clear model which handles this correlation. It is thus difficult to know whether a similarity between the image details at different resolutions is due to the property of the image itself or to the intrinsic redundancy of the representation. Further, this does not introduce any spatial orientation selectivity into the decomposition process. Davis ${ }^{6}$ introduced waveletbased fractal image coding, that was the generalization of fractal image coding in spatial domain. Davis showed that a total tree-based fractal compression method was very efficient in representing zero-tree-like structures and straight edges that have self-similarity. Still the large wavelet coefficients near edges are expensive to code. Current research concentrates on enabling wavelet coders to exploit the structure present in the wavelet coefficients along the edges. Successful coders perform variance of prediction across the frequency sub-bands using joint sub-band statistics ${ }^{7}$. Recently, a better approach, known as Fractal Zero-tree Wavelet (FZW) coder was proposed by Kim et al. ${ }^{8}$ based on the above concept. The basic

\footnotetext{
${ }^{*}$ Corresponding author (ksselvarajan@mail.vau.jfn.ac.lk)
} 
idea was to choose between the similar wavelet-domain structures that occur in EZW and tree-based fractal encoders by utilizing locally optimal, distortion-rate calculations.

In this paper a novel linear operator yielding a coder termed Self-Similar Wavelet High-Frequency Component (SWHFC) coder is proposed. The basic idea is to predict wavelet coefficients across the high-frequency sub-bands, enabling prediction from higher-frequency sub-band to lower high-frequency sub-band, based on the orientation, i.e. horizontal, vertical and diagonal. Obviously it leads to image compression. It is assumed that these frequency components posses scaled similarity across the frequency sub-band, based on the orientation. The linear operator uses weighting coefficients owing to the nature of the aliasing property of natural images.

Higher performance of this scheme is also dependent on encoding and decoding of truncation errors that occur during the encoding process. This is an inherent nature of the statistical estimation of wavelet coefficients across the frequency sub-bands. This has been efficiently handled by allocating some additional bits along with weighted coefficients. This scheme is computationally efficient. The trade-off between compression and distortion rate is optimal in the sense of other coders using wavelet coefficients.

This material is organised as follows: in this paper, first we describe the multiresolution representation of an image followed by similar frequency components that are evaluated in terms of EZW, based on the new prediction operator. A modified coding algorithm is proposed next based on the scheme of Selvarajan and Kodikara ${ }^{9}$. Experimental results which are obtained with this algorithm are evaluated with other known popular methods such as SPIHT and FZW, followed by the conclusions.

\section{Multiresolution representation of an image}

In this, the linear prediction operator is based on the decomposition of a function, $f$ (image) in the simplest wavelet basis, namely the Haar system. The Haar wavelets are compact, piecewise analytic, symmetric and orthogonal, but they lack in approximation property. Haar transform has enough smoothness to prove optimal rate of convergence in $L_{2}$ space than the smoother wavelet transforms. Using smoother wavelets may decrease the error by constant factor, because of their better performance in smooth regions of an image, but they will not achieve a better rate of convergence in $L_{2}$ space. Further, orthogonal transform preserves the energy, which is an essential requirement in nonlinear approximation of a function ${ }^{10}$. The univariate case is first considered and then it can be easily extended to multivariate case by tensor product of Haar wavelets.

Let $S:=S^{0}$ be the subspace of $L_{2}(\mathrm{R})$ that consists all piecewise constant functions with integer breakpoints: i.e. functions in $S$ are constants on each interval $(j, j+1)$, $j \in \mathrm{Z}$, then $S$ is a scale-invariant subspace of $L_{2}(\mathrm{R})$. A simple orthogonal basis for $S$ is given by the shifts of the function $\phi:=\chi_{[0,1]}$, i.e., $\phi_{j}(x)=\phi(x-j)$. By $\chi_{[0,1]}$ we denote the characteristic function of the interval $[0,1]$, namely.

$\chi_{[0,1]}=\left\{\begin{array}{lll}1 & \text { if } & x \in[0,1] \\ 0 & \text { if } & x \notin[0,1]\end{array}\right.$.

Now, each $f \in S$ has a unique representation such that,

$f(x)=\sum_{j \in Z}\left(c_{j}\right) \varphi_{j}(x), \quad\left(c_{j}\right) \in \ell_{2}(Z)$,

where $\ell_{2}(Z)$ is the set of square summable sequence and in $Z$, the set of integers. Then, approximation of a function $f \in L_{2}(\mathrm{R})$ is given by the orthogonal projection from $L_{2}$ (R) onto $S$ as,

$P_{o} f=\frac{1}{\left|I_{0, j}\right|} \int_{I_{0, j}} f \varphi_{j}(x) d x, \quad x \in I_{0, j}, \quad j \in Z$,

where $I_{0, j}$ denotes the interval of $(j, j+1), j \in Z$ Z. In other words, the first component in this decomposition is the average of, $f$, i.e., the projection onto the constant function $\varphi$,

$P_{0} f=\left\langle f, \varphi_{j}\right\rangle \varphi_{j}, \quad j \in Z$.

By dilation, we obtain sequence of spaces such that,

$S^{k}:=\left\{\varphi\left(2^{k}\right): \varphi \in S\right\}, \quad k \in Z$.

Now, $S^{k}$ is the space of piecewise constant function in $L_{2}(\mathrm{R})$ with breakpoints at the dyadic integers $j 2^{-k}, j \in Z$. The dyadic shifts $\varphi_{k, j}=2^{k / 2} \varphi\left(2^{k} .-j\right)$, with step size $j 2^{-k}, j \in Z$, of the function $\varphi\left(2^{k}\right)$ form an orthogonal basis for $S^{k}$. Then, the approximation is recursively refined into,

$P_{k} f=\sum_{j \in Z}\left\langle f, \varphi_{k, j}\right\rangle \varphi_{k, j}, \quad k \in Z$ 
That is, the averages of $f$ on the intervals $I_{k, j}=\left[j 2^{-k},(j+1) 2^{-k}\right)$. Clearly $P_{k} f$ is the $L_{2}(\mathrm{R})$ orthogonal projection of $f$ onto the space $S^{k}$ of piecewise constant functions on the intervals $I_{k, j}$, and $S^{k} \subset S^{k+1}, \quad k \in Z$. The limiting spaces,

$S^{\infty}=\overline{\bigcup_{k \in Z} S^{k}}$ and $\quad S^{-\infty}=\bigcap_{k \in Z} S^{k}$.

We claim that

$S^{\infty}=L_{2}(R)$ and $S^{\infty}=\{0\}$.

It can be easily shown that $P_{k} f \rightarrow f$ as $k \rightarrow \infty$. Consider the orthogonal complement projection $Q_{k}=P_{k} f-\mathrm{P}_{\mathrm{k}-1} f$. It follows that each $f \in L_{2}(R)$ can be represented by the series,

$f=\sum_{k \in Z}\left(P_{k}-P_{k-1}\right)=\sum_{k \in Z} Q_{k} f$.

Because the partial sums, $P_{n} f-P_{-n} f$, of this series tend to $f$ as $\mathrm{n} \rightarrow \infty$. The operator $Q_{k}$ is the orthogonal projector onto sup-space of $W^{k} \oplus S^{k}=S^{k+1}$. The space $W^{k}$ are dilates of the wavelet space

$W \oplus S^{0}=S^{1}$.

Since the spaces $W^{k}, k \in Z$, are mutually orthogonal, we have $W^{k} \perp W^{j}, k \neq j$, and equation (2) shows that $L_{2}(\mathrm{R})$ is the orthogonal direct sum of $W^{k}, k \in Z$ :

$L_{2}(R)=\oplus W^{k}, k \in Z$.

Clearly, the space $W_{k \in Z}^{k}$ is spanned by

$\psi_{k, j}=2^{k / 2} \psi\left(2^{k},-j\right), \quad j \in Z$

where $\psi$ takes the value 1 in $[0,1 / 2)$ and takes the value -1 in $[1 / 2,1)$ and 0 elsewhere. By dilation the function $\psi_{k, j}, j \in Z$ form a complete orthogonal system for $W^{k}$. Hence, orthogonal projection operator $Q_{k}$ of $L_{2}(\mathrm{R})$ onto $W^{k}$ by

$Q_{k} f=\sum_{j \in Z}\left\langle f, \psi_{k, j}\right\rangle \psi_{k, j}$.

Using this in equation (2), we have for any $f \in L_{2}(R)$ the decomposition

$f=\sum_{k \in Z} \sum_{j \in Z}\left\langle f, \psi_{k, j}\right\rangle \psi_{k, j}$.

Due to the above fact one has,

$\|f\|_{L_{2}(R)}=\left(\sum_{k \in Z}\left\|Q_{k} f\right\|_{L_{2(R)}}^{2}\right)^{1 / 2}=\|d(f)\|_{L_{2(Z)}}$.
This implies, small changes in $d(f)$ may cause only equally small changes in $f$ and vice versa, we have a particularly favourable digit representation of $f$.

While summability in equation (7) implies that the wavelet coefficients $d_{k, j}(f)$ eventually tend to zero - their size conveys local information about $f$. Since the wavelets $\psi_{k, j}$, are orthogonal to constants, we have

$\left|d_{k, j}(f)\right|=i n F_{c \in R}\left|f-c, \psi_{k, j}\right| \leq i n F_{c \in R}\|f-c\|_{L_{2}(R)} \leq 2^{-k}\left\|f^{\prime}\right\|_{L_{2}(R)}$.

Thus, $d_{k, j}(f)$ is small when $f$ is smooth on the support $I_{k, j}$ of $\psi_{k, j}$.

If all wavelet coefficients $d_{k, j}(f)$ were known, keeping the $N$ largest among them and replacing all the others by zero, would provide an approximation to $f$ involving only $N$ terms that minimizes, in view of equation (7), the $L_{2}$-error among the all other competing $N$ term approximation from the Haar system. This ability to provide sparse approximate representation of functions is a key feature of wavelet basis.

\section{The fast haar transform}

Computing wavelet coefficients directly through quadrature poses difficulties, since the support of low level wavelets is comparable to the domain, a sufficiently accurate quadrature would be quite expensive. A remedy is offered by the following strategy which can be used when the wavelet coefficients of interest have at most some highest level $K$ say. Given a function $f \in L_{2}(R)$, choose a large value of $\mathrm{n}$ and replace $f$ by $P_{\mathrm{n}} f$ with $P_{n}$, as before, the $L_{2}(\mathrm{R})$ projector onto $S^{n}$, the space of piecewise-constant function in $\mathrm{L}_{2}(\mathrm{R})$ with breakpoints at the dyadic integers $j 2^{-\mathrm{n}}, j \in Z$. Then we have (assume that $f$ has compact support)

$P_{n} f=\left(P_{n} f-P_{n-1} f\right)+\ldots . .+\left(P_{1} f-P_{0} f\right)+P_{0} f=P_{0} f+\sum_{k=0}^{n-1} Q_{k} f$.

which is a finite Haar decomposition, then we have

$Q_{k} f=\sum_{j \in Z} d_{k, j}(f) \psi_{k, j}, \quad d_{k, j}(f)=\left\langle f, \psi_{k, j}\right\rangle$.

and

$P_{k} f=\sum_{j \in z} c_{k, j}(f) \varphi_{k, j,} \quad c_{k, j}(f)=\left\langle f, \varphi_{k, j}\right\rangle$.

These coefficients are related to the integrals of $f$ over the intervals $I_{k, j}:=\left[j 2^{-k},(j+1) 2^{-k}\right)$ by,

$c_{k, j}=2^{k / 2} \int_{I_{k, j}} f d x$ and $d_{k, j}=2^{k / 2}\left[\int_{I_{k+1,2 j}} f d x-\int_{I_{k+1,2 j+1}} f d x\right]$. 
Therefore, if the coefficients $c_{k+1, j}, j \in Z$ are known, then

$c_{k, j}=\frac{1}{\sqrt{2}}\left(c_{k+1,2 j}+c_{k+1,2 j+1}\right)$ and $d_{k, j}=\frac{1}{\sqrt{2}}\left(c_{k+1,2 j}-c_{k+1,2 j+1}\right)$.

That is, coefficients are computed iteratively. Similarly, reconstruction of a function can be done iteratively. Now, suppose that we know the values $c_{0, j}, j \in Z$ and $d_{k, j,} j \in Z, k=0,1 \ldots \mathrm{n}-1$, and we wish to find $c_{n, j}$, i.e., to reconstruct $f$

$c_{k+1,2 j}=\frac{1}{\sqrt{2}}\left(c_{k, j}+d_{k, j}\right)$ and $c_{k+1,2 j+1}=\frac{1}{\sqrt{2}}\left(c_{k, j}-d_{k, j}\right)$.

Now, consider a function in some smooth spaces and suppose we know the values $c_{0, j}$ and $d_{k, j}$ itself, then the problem is how to reconstruct $f$. The solution is prediction of $d_{k, j}$ which gives us compression. This is the theme of this paper for which we have taken gray-scale images as the function to be compressed with the algorithm SWHFC.

Wavelet transform coding with piecewise constant approximation

Wavelet coefficients associated with high-frequency components depend on a small rough region located around the edge and not on overall smoothness of the picture. Conversely, smooth regions are less likely to be rippled off due to nearby edges. Moreover, equation (8) tells us that in region where $f$ has small derivative, the mesh (grid) size would be relatively coarse, while small intervals are encountered where $f$ varies strongly.

Consider projections of a function $f$ on two subspaces $W^{k}$ and $W^{k+1}$. These two projections isolate two different frequency sub-bands. From the previous fact, if function $Q_{k} f$ is rough in some region, then $Q_{k+1} f$ is also rough in the corresponding region. These wavelet coefficients of the high-frequency components represent the contours of an image. These contours show linear behaviour across the scales with respect to their orientation. This phenomenon is known as scaled similarity across the scales. In view of the above concept, coefficients at the resolution level $2^{-k}$ can be computed from the coefficients of the resolution level $2^{-(k+1)}$.

Let $f$ be a function in $L_{2}(\mathrm{R})$. Then, the function would be represented as

$f=\sum_{j \in Z} d_{k, j} \psi_{k, j} \quad d_{k, j}=\int f \psi_{k, j} \quad k=0,1, \ldots \ldots, n$.

Because of orthogonality we have

$d_{k, j}=\frac{1}{\sqrt{2}}\left(\int f \psi_{k+1,2 j}+\int f \psi_{k+1,2 j+1}\right)$.
The transformation is efficient in terms of computation but, it leaves residual correlation between highfrequency components, which is due to aliasing from the low-frequency components of the original image. Thus, the above does not apply for natural images. Wavelet spaces are series of function spaces that are highly uncorrelated from each other and are particularly suitable for the representation of signals and operators at different resolutions. Despite the de-correlation properties of the wavelet decomposition, it is apparent that coefficient with large magnitude tend to occur at the same relative locations in sub-bands at different scales. It holds when comparing sub-bands of the same orientation across the frequency sub-bands. In order to represent the relationship, consider the coefficient of coarser scale and corresponding coefficients of the finer scale with respect to their orientation. It is represented by the linear prediction as:

$d_{k, j}=\frac{1}{\sqrt{2}}\left(\omega_{k+1,2 j} \int f \psi_{k+1,2 j}+\omega_{k+1,2 j+1} \int f \psi_{k+1,2 j+1}\right)$.

where $\omega_{k}$ is the predicative coefficient between the frequency sub-band $2^{-k}$ and $2^{-(k+1)}$ respectively. The above is then represented as

$$
d_{k, j}=\frac{1}{\sqrt{2}} \sum_{j \in Z} \omega_{k, j} d_{k+1, j}, \quad k=0,1, \ldots \ldots, n
$$

where $d_{k+\mathrm{j}}$ be the wavelet coefficients. The predictive coefficient $\omega$ is calculated as to minimize the mean squared prediction error over the set of linear estimation defined in equation (1) as,

$\omega=\varepsilon\left(D . D^{T}\right)^{-1} \varepsilon(C . D)$

where $\varepsilon($.$) expected value of a random variable, C$ be the corresponding wavelet coefficient being estimated and $D$ be the vector of wavelet coefficients conditioning neighbour. It is proposed to calculate the $\omega$ by

$\omega_{k}=\frac{M \cdot C}{\sum_{j \in Z} d_{k+1, j}}$

where $M$ is the size of the unit interval of the frequency sub-band $2^{-(k+1)}$.

\section{Quad tree representation of wavelet coefficients}

Wavelet decomposition of a function $f \in L_{2}(R)$, approximates $f$ at several resolution levels, i.e., if $f \in L_{2}(R)$ then $P_{0} f$ of $f$ would be the best constant approximation and $P_{1} f$ is the best piecewise constant approximation on grid $2 \times 2$ of sub-squares $[0,1]^{2}$, similarly 
$P_{2} f$ of $f$ is the best piecewise constant approximation on $4 \times 4$ grid of sub-squares $[0,1]^{2}$. It is obvious that, smooth part of a function $f$ can be approximated with large subsquares and non smooth part of the function could be approximated with small sub-squares. For this reason each resolution level is considered on grid $2 \times 2$ and organised as quad-tree form and the quad tree is approximated by assigning weights to each nodes. Because of the dyadic nature of the wavelet decomposition, the 2-D wavelet transform is typically arranged in three sub-bands, corresponding to their orientation of the wavelet basis functions. Each sub-band can be organised into a quadtree, as described below.

In the quad-tree interpretation of the 2-D wavelet transform, each node $i$ is labeled with a wavelet coefficient $c_{k, j, \psi}{ }^{(k)}$, where the corresponding wavelet basis function $\psi^{(k)}$ has approximate support on a square, dyadic block $B_{i}$, in the image. The width of this block is given by $M=2^{-l} N$, where $l$ is the depth of node $i$ in the quad-tree and $N$ is the width of the pixels of the square image (assumed to be the power of two).

Except at the finest level, each node has four offspring representing $M / 2 \times M / 2$ dyadic blocks that combine to tile the same $M \mathrm{x} M$ image block as their parent. The objective is to code the finest level coefficients from which it estimates the next lower high-frequency subband components, in order to reduce the coding position information of each wavelet coefficient.

Proposition 1: Let $c_{l+1, \psi}(2 i+m, 2 j+n) \neq 0$ be the coefficient of highest-frequency components and $\omega_{m, n}$ be the weight of each edge that correspond to next lower high-frequency component coefficient in the same orientation, for levels $0<l<N$ and $0 \leq i, j<2^{k}$, then the coefficient of the next High-frequency component in the same orientation is estimated as:

$c_{l, \psi}\left(i^{l}, j^{l}\right)=\frac{1}{2} \omega_{m, n} \sum_{m=0}^{3} \sum_{n=0}^{3} c_{l+1, \psi}(2 i+m, 2 j+n)$.

where $\omega_{m, n}$ is the weight function in $\mathrm{R}$.

Proof : By above discussion

\section{Coding and decoding algorithm}

Images are transformed in three down sampling, fast Haar transform. Haar basis function carries out the mean value among the adjacent pixels that can decompose into the space-frequency. Exactly, this process repeats the finite difference operation by averaging the adjacent pixels exactly. Thus, it could be viewed as, higher- frequency components that are in quad-tree form $2 \times 2$ as the initial distribution and each having the weight $\omega$. With this terminology, the corresponding next lower high-frequency sub-band coefficients could be inferred from equation (18). This process could be repeated for finite number of times to infer the coefficients of highfrequency sub-band components. In coding process, high frequency coefficients are quantized to 6 bits including sign bit and weights of the nodes are rounded off to the next integer, in order to reduce the bit allocation for each weight, as such the difference of the values are stored along with the weight of each node. The weight and difference of the weight are quantized to 4 bits including sign bit. It facilitates to recover from the error during the prediction, as the result of truncation during the process.

\section{Encoding algorithm:}

(1) Given image is transformed using Haar orthogonal basis filter in three dyadic levels and wavelet coefficients are represented in Mallat form

(2) Coefficients are quantized by using nonlinear estimation

(3) Lower frequency sub-band is transmitted / stored

(4) Each, nonzero coefficients of highest frequency sub-bands are $(2 \times 2)$ and are coded with quad-tree address and transmitted/stored

4.1 Weighted coefficients are coded with their corresponding node address

4.2 Differences of the weighted coefficient values are stored along the weights

\section{Decoding algorithm:}

(1) Lower frequency component coefficients are placed in corresponding array position

(2) Highest frequency sub-bands coefficients are placed in the corresponding position of the array

(3) Next high frequency sub-bands are estimated recursively with weight of the nodes and the difference of values.

(4) Inverse wavelet transform is obtained to reconstruct the image with the Array

\section{RESULTS}

Our algorithm is named as SWHFC. Self-Similar Wavelet High-Frequency Component coding (SWHFC) is compared with other similar methods such as Fractal and EZW coding schemes in various domains, i.e., spatial domain and transformed domains (DCT and Wavelet), as it inherits those natures in this coding scheme. Among these the two methods known as SPIHT and FZW are 
superior in image compression technique. These methods use EZW and EZW \& fractal coding respectively. The results are available for 512 × 512 Lena, Babara and Goldhill 8 bits per pixel gray scale image as described by Taekon Kim et $a l^{8}$. The results are evaluated with Peak signal-to-noise ratio (PSNR) and Predicted peak signalto-noise ratio (PPSNR), the same weights adapted in. The traditional mean-squared-error and PSNR error measure are mainly focused on the pixel-by-pixel deference between the original and constructed image. Such metrics are improper for subjective quality assessment, since human perception is very sensitive to specific correlations between adjacent pixels. Recently, research on perceptual distortion measures that are matched to the human visual system (HVS) is vital in this context. The perceptual weighting for the sub-bands, which are

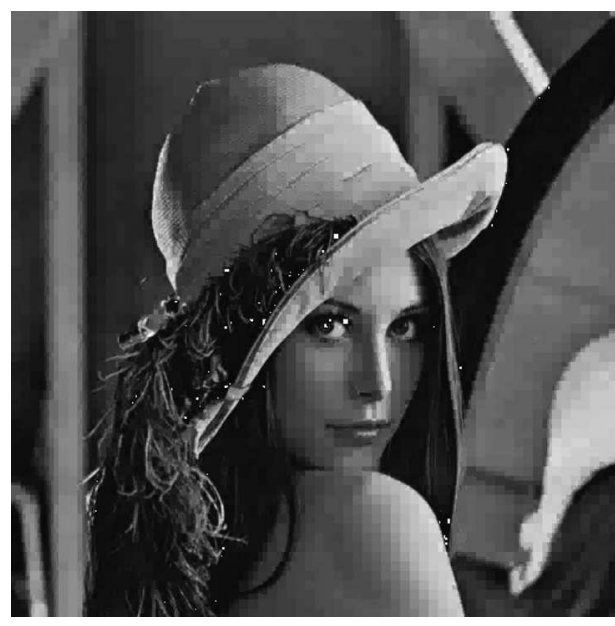

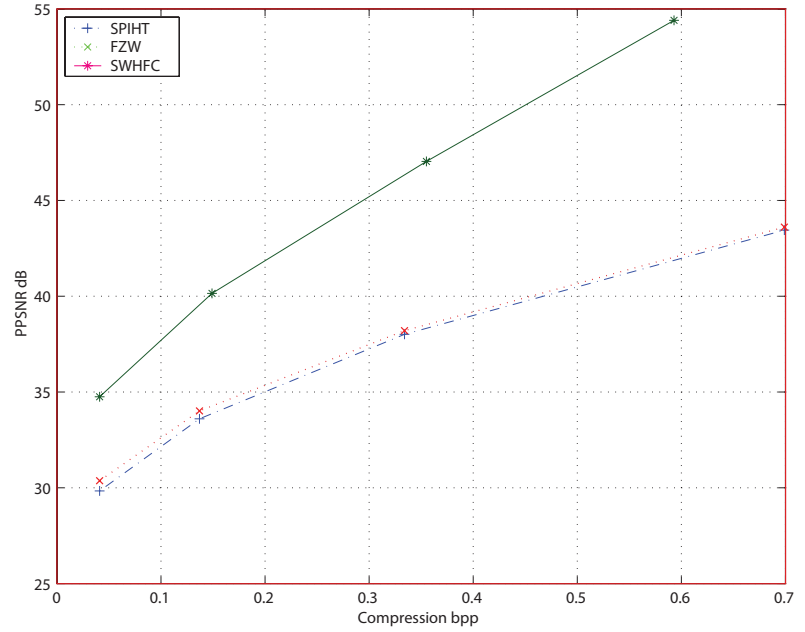

Figure 2: Distortion rate (PPSNR)

Lena 8 bits per pixel gray scale image.

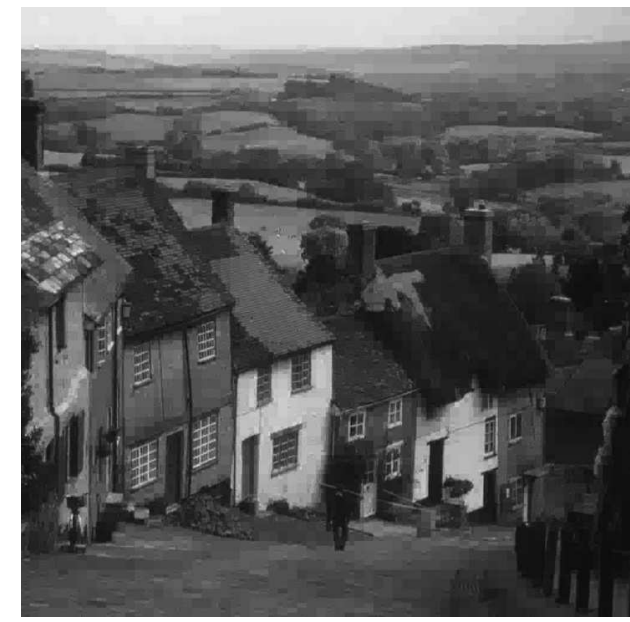

Figure 1: Reconstructed images 0.093bpp PPSNR 41.58dB and 0.102bpp PPSNR 41.70dB.

based on the "Just Noticeable Distortion" (JND), are used to increase perceptual image quality. A perceptual distortion measure that is commonly used is based on the Minkowski metric, and is known as Weighted Mean Squared Error (WMSE). This is given by

$W M S E=\frac{1}{N^{2}} \sum_{k} \sum_{i, j}\left(\frac{\omega_{k}(i, j)-w_{k}(i, j)}{t_{k}}\right)^{2}$,

where $\omega_{\mathrm{k}}(i, j)$ are the coordinates in the $k^{\text {th }}$ sub-band, $\omega_{\mathrm{k}}(i, j)$ are the corresponding predicted coefficients and $t_{k}$ is the weighting value for the $k^{\text {th }}$ sub-band. The order of the sub-band in an $l$-level decomposition is given by

$\{1.2, \ldots . M\}=\left\{s_{i}^{L L}, s_{i}^{H L}, s_{i}^{L H}, s_{i}^{H H}, s_{i-1}^{H L}, s_{i-1}^{L H}, s_{i-1}^{H H}, \ldots \ldots\right\}$,

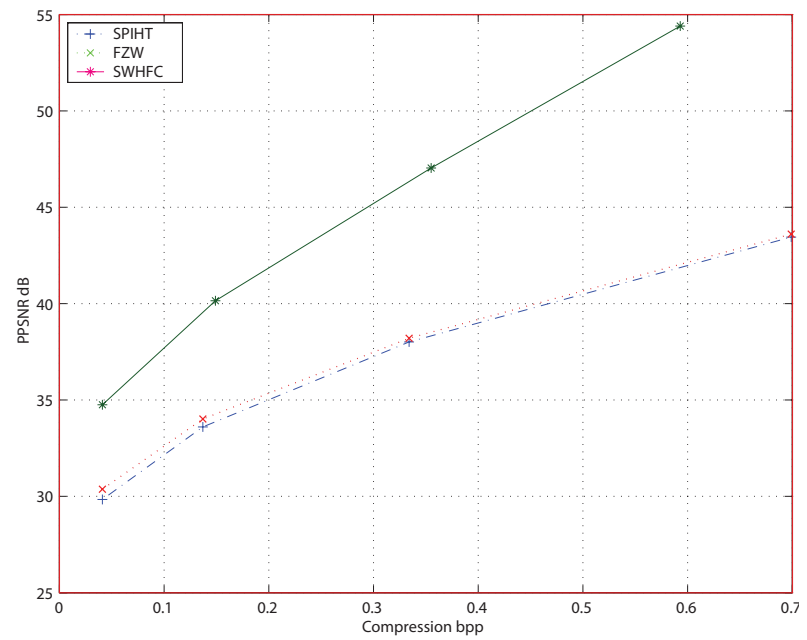

Figure 3: Distortion Rate (PPSNR)

Goldhill 8 bits per pixel gray scale image. 


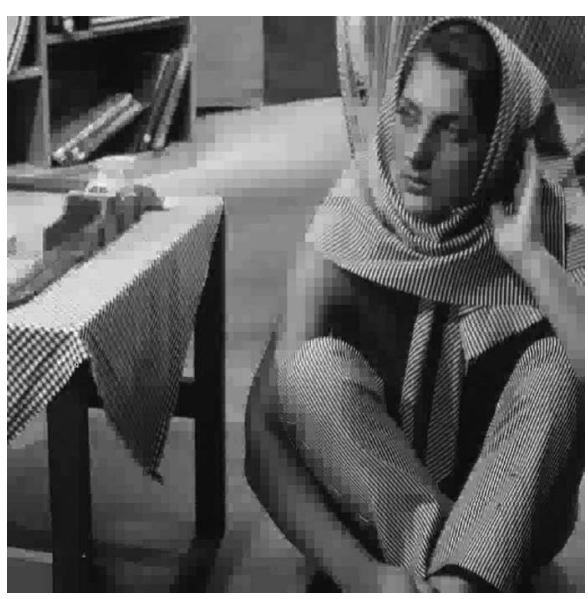

Reconstructed image, Barbara $0.149 \mathrm{bpp}$ PPSNR 40.15dB.

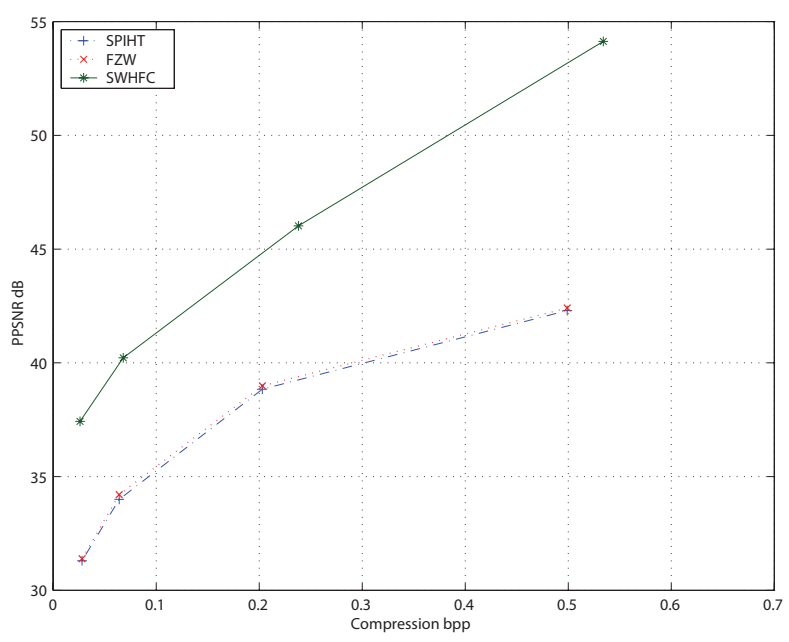

Distortion Rate (PPSNR). Barbara 8 bits per pixel gray scale image.

Figure 4

where $M=3 l+1$. In this experiment the following set of weights are adopted for the three-level decomposition as in.

$\left\{t_{k}\right\}=\{1.0,1.0,1.0,1.0,1.0,1.0,1.0,1.37,1.37,1.5\}$.

The results are given in terms of PSNR and perceptual PSNR (PPSNR), which is given by

$P P S N R=10 l o G_{10} \frac{255^{2}}{W M S E}$

The results are summarized in Figures 2, 3 and 4 for the above mentioned images. It clearly demonstrates the fact that SWHFC is superior in terms of compression and fidelity measure than the other methods. It should be noted that the error incurred in fidelity measure, in this scheme, is due to the quantization of high-frequency coefficients and predicative weighted coefficients. Wavelet aliasing and tiling effect are quite noticeable in high compression ratio as the consequence of nonlinear approximation of wavelet coefficients. The fidelity measure is closer to the nonlinear approximation. The other remarkable fact is that SWHFC is computationally highly efficient than other fractal coders or similar methods such as [1].

\section{CONCLUSION}

We have presented a linear prediction of wavelet coefficients across the frequency sub-bands with respect to their orientation. Implicitly predictions are along the edges of the image using the casual neighbour of the respective coefficients. Also, this predicts the coefficient magnitude and its sign. The Haar wavelets are known to provide local contrast values at each resolution and provide good ability to simulate HVS and strong attenuation at the edges of an image. So that Haar wavelet is used in this scheme. It is known, fractal coders are not competitive with state-of-the-art image coders. Trivially, this scheme implicitly inherits some aspects from fractal coders, but unlike fractal coders, SWHFC is efficient in terms of computational complexity. From the previous section, it is obvious SWHFC's performance is quite good in terms of the both fidelity measure.

There are a number of improvements that could be made in the implementation of SWHFC. The $\mathrm{L}_{1}$-norm combination of neighbouring magnitudes (15) could be replaced with an $\mathrm{L}_{\mathrm{p}}$-norm predictor, with $p$ chosen to optimize the coding gain. In addition the choice of wavelet decomposition in SWHFC could be altered with adaptive wavelet basis, in order to minimize the aliasing components from the lower frequency sub-band, where most of the energy is concentrated.

Recent developments in multi-scale methods have aimed to provide approximation and representation schemes that are similar to the Haar system in their hierarchical and algorithmic structure, with the use of more regular generating functions. There is an improvement that could be made in the implementation of the SWHFC. The transformation implemented in this scheme is arbitrary. It is possible to improve SWHFC by adapting some nonlinear transformation, which would yield fewer larger coefficients. It may lead to approximate such weighting coefficients across the frequency subbands.

\section{References}

1. Shapiro J. (1993). Embedded image coding using zerotrees of wavelet coefficients. IEEE Transaction on Signal 
Processing 41(12): 3445-3462.

2. Said A. \& Pearlman W.A. (1996). A new, fast and efficient image codec based on set partitioning in hierarchical trees. IEEE Transaction on Circuits and Systems for Video Technology 6(3): 243-250.

3. Cohen A., Dahmen W., Daubechies I. \& Devore R.A. (1999). Tree approximation and optimal encoding. IGPM Report 174, RWTH Aachen University, Templegraben, Aachen, Germany.

4. Cohen A., Daubechies I., Guleryns O. \& Orchard M. (2002). On the importance of combining wavelet based nonlinear approximation with coding strategies. IEEE Transaction of Information Theory 48(7): 1895-1921.

5. De Vore R.A. (1997). Nonlinear approximation. In: Acta Numerica (Ed. A. Iserles ). pp.261-283. Cambridge University Press, London, UK.

6. Rinaldo R. \& Calvagno G. (1995). Image coding by block prediction of multiresolution subimages. IEEE Transaction on Image Processing 4(7): 909-920.

7. Davis G. (1998). A wavelet-based analysis of fractal image compression. IEEE Transaction on Image Processing 7(2): 141-154.

8 Buccigrossi R.W. \& Simoncelli E.P. (1999). Image compression via joint statistical characterization in the wavelet domain. IEEE Transaction of Image Processing 8(12): 1688-1700.

9. Kim T., Dyck R.E.V. \& Miller D.J. (2002). Hybrid fractal zero-tree wavelet image coding. Signal Processing: Image Communication 17(4): 347-360.

10. Selvarajan S. \& Kodikara N.D. (2003).Image coding using the self-similarity of wavelet high-frequency components. In: Towards an ICT Enabled Society: Proceedings of the $5^{\text {th }}$ International Information Technology Conference (IITC) (Eds. V.K. Samaranayake, A.R. Weerasinghe \& P. Wimalaratne). pp. 83-90. University of Colombo, Colombo 03. 